\title{
A decade of mycophenolate mofetil for lupus nephritis: is the glass half-empty or half-full?
}

\author{
Dimitrios T Boumpas, ${ }^{1}$ George K Bertsias, \\ James E Balow?
}

The treatment of proliferative lupus nephritis (LN) can be staged as a period of intensive immunosuppressive therapy aimed at halting immunological injury (induction therapy) followed by a period of less aggressive maintenance therapy to consolidate the response. Maintenance therapy should be continued into the period of disease quiescence to decrease the number of flares and renal damage accrual. ${ }^{1}$ Randomised controlled trials (RCTs) have shown that adjunctive immunosuppressive agents are more effective than glucocorticoids alone in protecting against the risk of progression to end-stage renal disease (ESRD). Long-term ( $>10$ years) efficacy has been demonstrated only for cyclophosphamide (CY)-based regimens ${ }^{1-3}$; however, CY is associated with gonadal toxicity, which is both dose- and age-dependent. ${ }^{4}$ Mycophenolate mofetil (MMF), with little if any gonadal toxicity, has been examined as an alternative to CY for both induction and maintenance therapies. MMF has been shown in several RCTs to achieve comparable short-term rates of partial remission of $\mathrm{LN}$, but it remains to be proved whether it will ultimately be as effective as CY in pre-empting ESRD.

Irrespective of the choice of induction therapy, there is broad consensus that maintenance therapy must be well tolerated, reasonably affordable and have intrinsically low rates of toxicity. Currently, the two most common choices for maintenance therapy are MMF and

\footnotetext{
${ }^{1}$ Rheumatology, Clinical Immunology, and Allergy, University of Crete School of Medicine and Institute of Molecular Biology and Biotechnology, Heraklion, Greece

${ }^{2}$ National Institute of Diabetes and Digestive and Kidney Diseases, National Institutes of Health, Bethesda, Maryland, USA
}

Correspondence to Dimitrios T Boumpas, Rheumatology, Clinical Immunology, and Allergy, Institute of Molecular Biology and Biotechnology, University of Crete School of Medicine, 1 Voutes Street, Heraklion 71 500, Greece;

boumpasd@med.uoc.gr azathioprine (AZA). These two agents have distinct but similar mechanisms of action as purine antimetabolites. There is ongoing controversy about the objective evidence for the superiority of $\mathrm{MMF}$ over AZA as maintenance therapy in organ transplantation as well as in other immune-mediated diseases. ${ }^{5}$ As a result, some have argued that the lack of objective superiority of MMF on organ survival does not justify the 16-fold higher cost of MMF over AZA, although it is expected to be reduced after the patent expires at the end of this year. ${ }^{6}$ The fact that MMF appears to have some uniquely severe side effects-such as the Food and Drug Administration's Black Box warnings citing the risk of teratogenicity and progressive multifocal leucoencephalopathy-make it necessary to continue efforts to define the more favourable risk/benefit profiles of these two drugs for long-term maintenance therapy for numerous immunemediated diseases including LN.

In this issue of the journal, Houssiau et al ${ }^{6}$ report that MMF and AZA are indeed comparable as maintenance therapy in European patients with LN. In this investigator-initiated multicentre study (MAINTAIN), 105 patients with focal, diffuse or mixed proliferative LN (WHO class III-IV) received induction therapy with three intravenous pulses of methylprednisolone (MP) followed by oral glucocorticoids and six two-weekly intravenous pulses of $500 \mathrm{mg}$ CY. Patients were randomised at week 12 and received either AZA or $M M F$, irrespective of the magnitude of the renal response at the end of the intravenous CY treatment period (3 months). Parenthetically, one may argue that this is closer to clinical practice as substantive renal responses are relatively uncommon within the first 3-6 months. Analyses were by intent-to-treat and time to renal flare was the primary end point. Over a 3 -year period, the two groups receiving MMF or AZA maintenance therapies did not differ in time to renal flare, number of severe flares, renal remission or doubling of serum creatinine. Specifically, there were 13 renal flares (25\%) in the AZA group compared with 10 (19\%) in the MMF group; the serum creatinine doubled in four patients in the AZA group and in three in the MMF group. Eleven patients in the MMF group developed leucopenia compared with two in the AZA group. There was no significant difference in the prevalence of infections.

This study corroborates previous findings by Contreras et al which suggests that, following a short course with high-dose intravenous CY (7 monthly boluses of $0.5-1.0 \mathrm{~g} / \mathrm{m}^{2}$ body surface area to induce a nadir leucocyte count of $\geq 2000$ cells $/ \mathrm{mm}^{3}$ ), AZA and MMF are equally efficacious as maintenance regimens, but differs from the findings of the maintenance part of the Aspreva Lupus Management Study (ALMS). ${ }^{8}$ In this study, the largest in the history of treatment of LN, 370 patients with class III-V LN were randomised to receive open-label induction therapy with MMF (target dosage $3 \mathrm{~g} /$ day) or intravenous CY (0.5$1.0 \mathrm{~g} / \mathrm{m}^{2}$ in monthly pulses) in combination with corticosteroids (tapered from a maximum starting dose of $60 \mathrm{mg} /$ day) for 24 weeks. The primary efficacy end point was treatment response defined as decreased proteinuria and stabilised (within $25 \%$ of baseline) or improved serum creatinine. Patients achieving response or complete remission (defined as normalisation of all parameters) were subsequently re-randomised to doubleblind placebo-controlled maintenance treatment with MMF or AZA, both combined with corticosteroids. For the induction phase, the study did not detect a significantly different response rate between the two groups. For the maintenance phase, the primary end point was time to treatment failure (defined as either flares, need to intensify therapy, doubling of serum creatinine or death). Preliminary results from the latter presented at the 9th International Congress on Systemic Lupus Erythematosus in Vancouver, Canada in June 2010 showed a failure rate of $32 \%$ in the AZA group compared with $16 \%$ in the MMF group at 3 years after successful induction therapy during the first part of the study with either MMF or CY. ${ }^{10}$ While eagerly awaiting the full report of the ALMS trial to establish whether these differences reflect the larger number of patients (105 in MAINTAIN vs 227 in ALMS), the 
outcomes used (single in MAINTAIN vs composite in ALMS), the ethnic background of the treated patients or differences in the dose of corticosteroids and the duration of maintenance treatment, the MAINTAIN study clearly demonstrates equivalence between AZA and MMF in European patients.

The reporting of the MAINTAIN and ALMS trials marks the completion of a decade of MMF trials. Table 1 summarises the key features of RCT studies in LN during this decade, ${ }^{7} 811-18$ including rates of 'response' at $\sim 6$ months and 'relapse' at $\sim 3$ years for different therapies. One can see that there is a wide range in the rates of these outcomes, making it difficult to interpret emerging studies even when there is statistical significance within a particular study.
This analysis certainly emphasises the need for long-term data with hard renal outcomes such as ESRD or its surrogate doubling of serum creatinine. Are we at the end of this decade in a better position to objectively evaluate the efficacy of MMF in LN? Is the glass 'half-empty' or 'half-full'? What are the ramifications of these trials for the modern treatment of LN?

We believe that the glass is certainly 'half-full' and these studies have added a major new drug to the therapeutic armamentarium of LN. At the same time, as in the case with every single drug used in LN (including CY), the glass is not 'full' as these studies have highlighted important shortcomings of MMF such as failure of a significant number of patients to reach a complete response and lack of long-term data. Although there is no head-to-head comparison between MMF and AZA for induction of remission in $\mathrm{LN}$ and in spite of the lack of long-term data for MMF, it is reasonable to assume that MMF could be the drug of first choice for the induction of remission in moderately severe $\mathrm{LN}$ (table 2), especially in black and Hispanic individuals. This is based both on the basis of better quality data for MMF originating from larger multicentre trials around the world and clinical experience whereby some patients refractory to CY respond to $\mathrm{MMF}$, something unusual for AZA. In our opinion, whether MMF is superior in these subsets remains to be confirmed in future trials. This should not be perceived as abandoning AZA for $\mathrm{LN}$ altogether as recent studies have shown efficacy when combined with

Table 1 Efficacy of induction and maintenance therapies in PLN

\begin{tabular}{|c|c|c|c|c|c|c|c|c|}
\hline \multirow[b]{2}{*}{ Induction studies* } & \multirow[b]{2}{*}{$\mathbf{N}$} & \multicolumn{5}{|c|}{ Baseline patient characteristics } & \multicolumn{2}{|c|}{ Response (6 months) } \\
\hline & & Black/Hispanic (\%) & PLN (\%) & $\mathrm{mSCr}$ (mg/dl) & $\mathrm{mUp} / \mathrm{Ucr}(\mathrm{g} / \mathrm{g})$ & mSAlb (mg/dl) & CY (\%) & MMF (\%) \\
\hline Chan et al ${ }^{11}$ & 41 & $0 / 0$ & 100 & 1.2 & 4.7 & 2.8 & 90 & 95 \\
\hline Houssiau et al ${ }^{12}$ & 90 & $9 / 0$ & 100 & 1.2 & 3.0 & 3.0 & $\sim 60$ & NA \\
\hline Ginzler et $a^{13}$ & 140 & $56 / 20$ & 70 & 1.1 & 4.3 & 2.8 & 30 & 52 \\
\hline Ong et $a l^{14}$ & 24 & $0 / 0$ & 100 & 1.1 & 2.5 & 2.9 & 52 & 58 \\
\hline Grootschölten et a/ ${ }^{15}$ & 50 & $20 / 0$ & 100 & 0.7 & 3.9 & - & 75 & NA \\
\hline Lu et a/ ${ }^{16}$ & 213 & $0 / 0$ & 100 & 1.3 & 4.7 & 2.6 & NA & 83 \\
\hline \multirow[t]{2}{*}{ Appel et $a /^{8}$} & 370 & $12 / 35$ & 84 & 1.1 & 4.1 & - & 53 & 56 \\
\hline & & \multicolumn{5}{|c|}{ Baseline patient characteristics } & \multicolumn{2}{|c|}{ Flares (3 years) } \\
\hline Maintenance studies $†$ & $\mathbf{N}$ & Black/Hispanic (\%) & PLN (\%) & $\mathrm{mSCr}$ (mg/dl) & mUp/Ucr $(g / g)$ & mSAlb (mg/dl) & AZA (\%) & MMF (\%) \\
\hline Houssiau et a/ ${ }^{12}$ & 83 & $9 / 0$ & 100 & 1.2 & 3.0 & 3.0 & 25 & $\mathrm{NA}$ \\
\hline Contreras et $\mathrm{al}^{7}$ & 39 & $46 / 49$ & 98 & 1.6 & 5.1 & 2.7 & 43 & 23 \\
\hline Chan et al ${ }^{11}$ & 62 & $0 / 0$ & 100 & 1.2 & 4.8 & 2.8 & 15 & 35 \\
\hline Grootscholten et a/ ${ }^{15}$ & 37 & $20 / 0$ & 100 & 0.7 & 3.9 & - & 17 & NA \\
\hline Moroni et al ${ }^{18}$ & 33 & $0 / 0$ & 88 & 0.9 & 2.5 & - & 24 & NA \\
\hline Houssiau et $a l^{6}$ & 105 & $12 / 0$ & 89 & 1.0 & 3.3 & 3.0 & 22 & 19 \\
\hline Wofsy et al ${ }^{10}$ & 227 & $12 / 35$ & 84 & 1.1 & 4.1 & - & 32 & 16 \\
\hline
\end{tabular}

Results from recent (2000-10) RCTs demonstrate significant heterogeneity in treatment outcomes.

${ }^{*}$ Response defined as at least $50 \%$ decrease in proteinuria and to levels $<3.5 \mathrm{~g} / 24 \mathrm{~h}$.

tFlares correspond mostly to doubling of proteinuria.

$\mathrm{AZA}$, azathioprine; CY, cyclophosphamide; MMF, mycophenolate mofetil; mSAlb, mean serum albumin; $\mathrm{mSCr}$, mean serum creatinine; mUp/Ucr, mean urine protein-to-creatinine ratio; NA, not applicable; PLN, proliferative lupus nephritis.

Table 2 Markers of severity and risk for end-stage renal disease (ESRD) in biopsy-proven proliferative lupus nephritis

\begin{tabular}{|c|c|c|c|c|}
\hline & Nephritis class & Adverse renal histology* & $\begin{array}{l}\text { Impaired renal function and/or severe } \\
\text { proteinuriat }\end{array}$ & $\begin{array}{l}\text { Response to } \\
\text { immunosuppressive therapy } \neq\end{array}$ \\
\hline Mild disease & Focal proliferative & No & No & NA \\
\hline \multirow[t]{3}{*}{ Moderately severe disease } & Focal proliferative & No & No & Partial or no response \\
\hline & Focal proliferative & Either present & & NA \\
\hline & Diffuse proliferative & No & No & NA \\
\hline \multirow[t]{4}{*}{ Severe disease } & $\begin{array}{l}\text { Diffuse proliferative classified as } \\
\text { moderately severe }\end{array}$ & NA & NA & Partial or no response \\
\hline & Focal or diffuse proliferative & Yes & Yes & NA \\
\hline & Mixed proliferative and membranous & NA & NA & NA \\
\hline & Rapidly progressive glomerulonephritis & NA & NA & NA \\
\hline
\end{tabular}

This classification is based upon findings of longitudinal observational studies, retrospective analyses of RCTs or clinical cohorts with regard to histological, clinical or serological factors associated with development of ESRD. The prognostic value of some of these factors in the context of mycophenolate mofetil treatment needs to be defined.

${ }^{*}$ Crescents and/or fibrinoid necrosis affecting $>25 \%$ of glomeruli; glomerular sclerosis, tubular atrophy or chronicity index $>4$; or chronicity index $>3$ and activity index $>10$.

tIncrease in serum creatinine or reduction in estimated glomerular filtration rate (calculated by the Cockcroft-Gault or the Modification of Diet in Renal Disease formula) by $>25 \%$;

proteinuria $>4 \mathrm{~g} / 24 \mathrm{~h}$.

$\ddagger$ Assessed after 6 months of therapy. ${ }^{19-21}$

NA, not applicable. 
pulsed intravenous $\mathrm{MP}$, albeit inferior to that of CY. ${ }^{15}$ AZA may be used both as an induction therapy in milder cases of $\mathrm{LN}$ in white patients and as maintenance therapy in most patients except probably in those with severe disease and certain racial or ethnic characteristics. Failure to achieve a complete response after the initial 6 months of treatment should precipitate discussions for switching to intravenous CY, preferably in combination with intravenous MP for the first 3-6 months. ${ }^{15}$

For patients with severe LN, the best available data support the combined use of pulses of intravenous MP and intravenous CY at least for the first 6 months, ${ }^{19}$ with AZA or MMF used as maintenance therapy. Although this regimen has not been tested formally, we have shown that, in patients with severe LN, a long course of intravenous CY (15 pulses) is effective in preserving renal function in the long term. ${ }^{13}$ Thus, based upon clinical experience and data from RCTs, we can assume that, in patients with severe LN who achieve a complete renal response, switching to maintenance therapy with AZA or MMF is a reasonable option. For maintenance therapy, MMF or AZA could be used based on availability and potential for pregnancy as MMF is associated with an increased risk of spontaneous abortion and fetal malformation. Because of the significant difference in the cost between the two drugs, patients with mild-to-moderate LN could first be treated with AZA, especially white individuals. In contrast to proliferative $\mathrm{LN}$, the data for MMF on membranous LN are limited to small retrospective cohorts with some studies demonstrating efficacy while others have failed to do so.

The use of intravenous MP pulses in current induction treatment protocols cannot be overemphasised. There are circumstantial data to support the use of 1-3 intravenous MP pulses, especially for patients with moderate or severe nephritis. In addition to expediting remission, intravenous MP pulses may also allow for the use of lower doses of glucocorticoids at the early phases of the induction period. Irrespective of the treatment used, adherence to the National Kidney Foundation-Kidney Disease Outcomes Quality Initiative (NKF-KDOOI) guidelines for the management of chronic kidney disease (accessible at www.kidney. org) is of paramount importance.

Where do these trials leave us at the end of this decade? While the excitement and anticipation with new agents is understandable, premature declaration of superiority or rushing into discrediting old treatments that have served patients well need to be avoided in the future. At the same time, the scientific and clinical community, regulatory agencies and industry need not lose sight of the lifelong course of the disease. Only long-term follow-up (>10 years) by means of registries or organised longterm observational studies will allow the full assessment of the newer agents and provide reasonable assurances to patients and physicians of their overall safety and efficacy.

Provenance and peer review Commissioned; externally peer reviewed.

Accepted 12 October 2010

Ann Rheum Dis 2010;69:2059-2061

doi:10.1136/ard.2010.139683

\section{REFERENCES}

1. Boumpas DT, Austin HA, 3rd, Vaughn EM, et al. Controlled trial of pulse methylprednisolone versus two regimens of pulse cyclophosphamide in severe lupus nephritis. Lancet 1992;340:741-5.

2. Austin HA, 3rd, Klippel JH, Balow JE, et al. Therapy of lupus nephritis. Controlled trial of prednisone and cytotoxic drugs. N Eng/ J Med 1986;314:614-19.

3. Bertsias G, loannidis JP, Boletis J, et al. EULAR recommendations for the management of systemic lupus erythematosus. Report of a Task Force of the EULAR Standing Committee for International Clinical Studies Including Therapeutics. Ann Rheum Dis 2008;67:195-205.

4. Boumpas DT, Austin HA, 3rd, Vaughan EM, et al. Risk for sustained amenorrhea in patients with systemic lupus erythematosus receiving intermittent pulse cyclophosphamide therapy. Ann Intern Med 1993;119:366-9.

5. Cravedi P, Perna A, Ruggenenti P, et al. Mycophenolate mofetil versus azathioprine in organ transplantation. Am J Transplant 2009;9:2856-7.

6. Houssiau FA, D'Cruz D, Sangle S, et al. Azathioprine versus mycophenolate mofetil for long-term immunosuppression in lupus nephritis: results from the MAINTAIN Nephritis Trial. Ann Rheum Dis 2010;69:2083-89.
7. Contreras G, Pardo V, Leclercq B, et al. Sequential therapies for proliferative lupus nephritis. N Engl J Med 2004;350:971-80.

8. Appel GB, Contreras G, Dooley MA, et al. Mycophenolate mofetil versus cyclophosphamide for induction treatment of lupus nephritis. J Am Soc Nephrol 2009;20:1103-12.

9. Sinclair A, Appel G, Dooley MA, et al. Mycophenolate mofetil as induction and maintenance therapy for lupus nephritis: rationale and protocol for the randomized, controlled Aspreva Lupus Management Study (ALMS). Lupus 2007;16:972-80.

10. Wofsy D, Appel GB, Dooley MA, et al. Aspreva Lupus Management Study maintenance results. Lupus 2010;19:S27.

11. Chan TM, Li FK, Tang CS, et al. Efficacy of mycophenolate mofetil in patients with diffuse proliferative lupus nephritis. Hong KongGuangzhou Nephrology Study Group. N Engl J Med 2000;343:1156-62.

12. Houssiau FA, Vasconcelos C, D'Cruz D, et al. Immunosuppressive therapy in lupus nephritis: the Euro-Lupus Nephritis Trial, a randomized trial of low-dose versus high-dose intravenous cyclophosphamide. Arthritis Rheum 2002; 46:2121-31

13. Ginzler EM, Dooley MA, Aranow C, et al. Mycophenolate mofetil or intravenous cyclophosphamide for lupus nephritis. N Engl J Med 2005;353:2219-28.

14. Ong LM, Hooi LS, Lim TO, et al. Randomized controlled trial of pulse intravenous cyclophosphamide versus mycophenolate mofetil in the induction therapy of proliferative lupus nephritis. Nephrology (Carlton) 2005;10:504-10.

15. Grootscholten C, Ligtenberg G, Hagen EC, et al. Azathioprine/methylprednisolone versus cyclophosphamide in proliferative lupus nephritis. A randomized controlled trial. Kidney Int 2006;70:732-42.

16. Lu F, Tu Y, Peng $X$, et al. A prospective multicentre study of mycophenolate mofetil combined with prednisolone as induction therapy in 213 patients with active lupus nephritis. Lupus 2008;17:622-9.

17. Chan TM, Tse KC, Tang CS, et al. Long-term study of mycophenolate mofetil as continuous induction and maintenance treatment for diffuse proliferative lupus nephritis. J Am Soc Nephrol 2005;16:1076-84.

18. Moroni G, Doria A, Mosca M, et al. A randomized pilot trial comparing cyclosporine and azathioprine for maintenance therapy in diffuse lupus nephritis over four years. Clin J Am Soc Nephrol 2006;1:925-32.

19. Gordon C, Jayne D, Pusey C, et al. European consensus statement on the terminology used in the management of lupus glomerulonephritis. Lupus 2009;18:257-63.

20. Bertsias G, Boumpas DT. Update on the management of lupus nephritis: let the treatment fit the patient. Nat Clin Pract Rheumatol 2008;4:464-72.

21. Ponticelli C, Glassock RJ, Moroni G. Induction and maintenance therapy in proliferative lupus nephritis. J Nephrol 2010;23:9-16. 